\title{
SUBSÍDIOS PARA A ASSISTÊNCIA DE ENFERMAGEM A PACIENTES COM VALVOPATIA MITRAL
}

\author{
Karim Mitie Kubo \\ Roberta Cunha Rodrigues Colombo ${ }^{2}$ \\ Maria Cecilia Bueno Jayme Gallani? \\ Rachel Noronha ${ }^{3}$
}

Kubo KM, Colombo RCR, Gallani MCBJ, Noronha R. Subsídios para a assistência de enfermagem a pacientes com valvopatia mitral. Rev Latinoam Enfermagem 2001 maio; 9(3):33-42.

Este estudo teve por finalidade caracterizar o perfil do paciente valvopata mitral, identificar seu conhecimento sobre a doença e levantar as principais dificuldades enfrentadas no seu dia-a-dia. Os dados foram obtidos através de entrevista semi-estruturada aplicada a 29 pacientes atendidos no ambulatório de um hospital universitário. Os dados foram analisados através da técnica de Análise de Conteúdo. Os resultados permitiram conhecer o problema sob a perspectiva do indivíduo e de sua família, o que constitui elemento subsidiador crítico para a elaboração de uma proposta de assistência de enfermagem, com enfoque educacional.

UNITERMOS: cuidados de enfermagem, valva mitral, educação do paciente

\section{MITRAL VALVE DYSFUNCTION: SUBSDY TO ELABORATE A PROPOSAL OF NURSING CARE FOR OUTPATIENTS}

The purpose of this study was to define: the profile of patients with mitral valve dysfunction (stenosis and/or insufficiency) who were assisted at the University Outpatients' Clinic, the knowledge that these patients had concerning their disorder and the main difficulties and limitations resulting from it. The necessary data were obtained by interviewing 29 patients from November 1997 to February 1998. An analysis was conducted through the Content Analysis Technique. The results obtained helped to understand the problem under the individual's and his family's viewpoint, which helped to elaborate a nursing care program with an educational focus.

KEY WORDS: mitral valve, health education, nursing care

\section{APORTES PARA LA ATENCIÓN DE ENFERMERÍA A PACIENTES CON VALVULOPATÍA MITRAL}

Este estudio tuvo como finalidad caracterizar el perfil del paciente con valvulopatía mitral identificar su conocimiento sobre la enfermedad y levantar las principales dificultades enfrentadas en su día a día. Los datos fueron obtenidos a través de entrevista semiestructurada aplicada a 29 pacientes atendidos en la consulta externa de un Hospital Universitario. Los datos fueron analizados a través de la técnica de Análisis de Contenido. Los resultados encontrados nos permitieron conocer el problema sobre la perspectiva del individuo y su familia, lo que constituyó la base para la elaboración de una propuesta de atención de enfermería con enfoque educativo.

TÉRMINOS CLAVES: válvula mitral, cuidado de enfermería, educación en salud

\footnotetext{
${ }^{1}$ Enfermeira do Instituto do Coração (InCor) da Faculdade de Medicina da Universidade de São Paulo

${ }^{2}$ Enfermeira, Doutor em Enfermagem, Professor Colaborador; ${ }^{3}$ Enfermeira, Doutor em Educação, Professor Assistente. Curso de Enfermagem da FCMUNICAMP. E-mail: mcbjgallani@neuro.med.br
} 


\section{INTRODUÇÃO}

As doenças cardiovasculares, no contexto da saúde mundial, destacam-se pelos seus elevados índices de morbidade e mortalidade.

No Brasil, as estatísticas que definem a magnitude do problema são estarrecedoras: 300.000 brasileiros são vítimas dessas doenças ao ano. Além de serem a maior causa de óbito em todo o país, independentemente da faixa etária, as enfermidades cardiovasculares são também as que acarretam maior ônus ao sistema de saúde ${ }^{(2)}$. Embora exista uma tendência de queda na taxa de mortalidade por doenças cardiovasculares, tanto em homens, como em mulheres, conforme apontam os dados de um estudo recente desenvolvido no Estado de São Paulo ${ }^{(3)}$, estas doenças ainda permanecem como a principal causa de mortalidade em nosso meio.

Neste conjunto de doenças do aparelho circulatório, embora preponderem em prevalência a doença isquêmica do coração, as doenças cerebrovasculares e a insuficiência cardíaca, encontramos também as valvopatias, principalmente aquelas de origem reumática. $\mathrm{Na}$ América Latina, nos locais onde há registro de febre reumática aguda, a incidência na faixa etária dos 5 aos 15 anos é, em média, 0,28 a 0,41 por 1000 - valor 3 a 4 vezes maior em relação ao encontrado nos Estados Unidos e em outros países ${ }^{(4)}$.

A valvopatia reumática, com destaque para o acometimento da valva mitral, permanece como a doença cardíaca mais comumente adquirida em todo o mundo e a principal causa de morte cardiovascular, durante as primeiras cinco décadas de vida, em países desenvolvidos ${ }^{(5-6)}$. Portanto, a valvopatia mitral, seja por estenose e/ou insuficiência, continua sendo um importante problema de saúde, apesar dos avanços obtidos na abordagem diagnóstica e terapêutica.

As diferentes disfunções do aparelho valvar mitral, apesar de possuírem suas especificidades, acabam por acarretar mudanças no estilo de vida de seus portadores, decorrentes das limitações fisiológicas, psicológicas e sociais impostas pela doença, a curto, médio e longo prazo. Estas limitações que estão relacionadas, principalmente, com atividades diárias (físicas e de trabalho) ${ }^{(7)}$, parecem interferir na qualidade de vida destes indivíduos.

As valvopatias crônicas cursam, na maior parte das vezes, com longos períodos de tratamento clínico e é geralmente a ineficácia da terapêutica clínica, a partir de uma determinada fase da evolução natural da doença, que determina a necessidade de intervenção cirúrgica $^{(8)}$.

Assim, verifica-se que o período compreendido entre 0 diagnóstico médico de uma valvopatia mitral até a indicação de uma intervenção cirúrgica é muito variável e que durante este período o indivíduo já experimenta sintomas que podem alterar significativamente o seu estilo de vida.
A convivência com os pacientes portadores de valvopatia mitral, atendidos no Ambulatório do Serviço de Cardiologia do Hospital de Clínicas da Universidade Estadual de Campinas (HC) UNICAMP), permitiu constatar que o serviço não contava com a participação efetiva do profissional de enfermagem na orientação destes pacientes, principalmente no que se refere ao auxílio a estes indivíduos na adoção de mudanças em seu estilo de vida, necessárias para adaptação às novas condições impostas pela valvopatia. Tal constatação levou-nos a refletir sobre a necessidade de uma assistência de enfermagem que fosse peculiar a esses indivíduos e os auxiliasse no processo de adaptação à doença, com melhora da sua qualidade de vida. Os recursos disponíveis na literatura para guiar o enfermeiro no cuidado de pacientes com valvopatias estão concentrados na fisiopatologia da doença, o que determina uma lacuna muito grande na compreensão global do indivíduo. Para o planejamento mais adequado dos cuidados de enfermagem, é importante considerar não apenas os aspectos fisiológicos, mas também aqueles psicológicos e sociais da doença, incluindo 0 significado dessa doença para o indivíduo e para aqueles com quem ele convive ${ }^{(19)}$.

0 Ambulatório de Cardiologia do HC-UNICAMP atende, atualmente, um número significativo de pacientes valvopatas, aos quais pode e deve ser direcionada uma assistência de enfermagem específica. Assim, o levantamento do grau de conhecimento desses sujeitos sobre sua doença, das principais dificuldades que eles enfrentam no dia-a-dia, pareceu-nos ser o primeiro e indispensável passo a ser realizado, para que se possa dar seqüência à estruturação dessa assistência.

Desse modo, este estudo teve por OBJETIVOS: traçar o perfil dos pacientes com Estenose Mitral (EMi) e Insuficiência Mitral (IMi) atendidos no Ambulatório de Cardiologia do Hospital de Clínicas da Universidade Estadual de Campinas (HC/UNICAMP); levantar o conhecimento do valvopata sobre sua doença e identificar as principais dificuldades enfrentadas por estes pacientes no seu cotidiano, em relação à doença e ao tratamento.

Com a finalidade de fundamentar este estudo, optamos por fazer algumas considerações com relação à etiologia, fisiopatologia, repercussões clínicas e principais intervenções terapêuticas na valvopatia mitral (estenose e insuficiência), conforme descritas a seguir: "Estenose Mitral (EMi) é a condição na qual o comprometimento da valva mitral prejudica o escoamento de sangue, do átrio esquerdo (AE) para o ventrículo esquerdo (VE), durante a diástole, devido ao espessamento dos folhetos valvares e das cordoalhas tendíneas e seu concomitante encurtamento, o que resultará na diminuição da área valvar e, conseqüentemente, aumento na pressão atrial esquerda e hipertensão pulmonar" ${ }^{\text {(7, 9- }}$ 11). "A superfície valvar mitral normal varia entre $4 \mathrm{a} 6 \mathrm{~cm}^{2}$. Quando 
reduz-se a aproximadamente $2 \mathrm{~cm}^{2}$ a EMi pode ser classificada como estenose moderada, na qual observa-se um gradiente de pressão para que o sangue possa fluir do AE para o VE. Quando esta superfície reduz-se a $1 \mathrm{~cm}$, considerado como EMi severa, há necessidade de um gradiente de pressão de aproximadamente 20 $\mathrm{mm} \mathrm{Hg}$ para manter o débito cardíaco normal em repouso. 0 aumento na pressão atrial esquerda culminará com a elevação das pressões venosa e capilar pulmonar, ocasionando dispnéia aos esforços ${ }^{(12)}$. A dilatação atrial por sua vez, serve como substrato anatômico para desencadeamento da fibrilação atrial. Tal distúrbio do ritmo cardíaco é responsável por significativa debilidade hemodinâmica dos pacientes com doença valvar mitral"(13). "Uma pequena parcela dos pacientes (15\%) relatam precordialgia, que pode ser devida à hipertensão ventricular direita; pode ocorrer também, hemoptise, como resultado da rotura das veias brônquicas, em decorrência do aumento súbito da pressão atrial. Também têm sido descritos episódios de tromboembolismo, principalmente em pacientes com fibrilação atrial ${ }^{(12)}$. A fibrilação atrial é conhecida como fator de risco associado ao embolismo sistêmico, quer esteja associada ou não à estenose mitral reumática, pois predispõe o átrio esquerdo à formação de trombos ${ }^{(14)}$. Nos pacientes com EMi severa e insuficiência ventricular direita, observam-se sinais de hipertensão venosa sistêmica (edema, ascite, hepatomegalia, entre outros)" ${ }^{\prime(9)}$. "A Insuficiência Mitral (IMi) consiste na incompetência da válvula mitral, que se torna incapaz de impedir que o sangue reflua do ventrículo esquerdo ao átrio esquerdo, durante a sístole ventricular $^{(10)}$. Anormalidades em quaisquer das estruturas do aparelho valvar mitral podem causar $\mathrm{IMi}$, que pode ser aguda ou crônica"(12). "A magnitude das anormalidades hemodinâmicas impostas às câmaras esquerdas é influenciada pela natureza crônica ou aguda do distúrbio valvar, além do estado funcional prévio do VE"(15). "Como resultado das repercussões hemodinâmicas, verificase aumento na pressão atrial esquerda (devido ao volume de sangue regurgitante do VE e o afluxo de sangue das veias pulmonares) e concomitante distensão atrial, o que resultará em uma contração atrial efetiva. Esta condição irá contribuir para posterior dilatação e hipertrofia do $\mathrm{AE}$ (mecanismo compensatório do AE). As alterações no átrio acarretam aumento do volume diastólico e pressão do VE, o que irá garantir a ejeção ventricular esquerda. Porém, o aumento do volume e pressão do VE acaba por determinar dilatação e hipertrofia desta câmara (mecanismo compensatório do VE). A compensação do quadro dependerá, portanto, da magnitude do afluxo e da complacência do AE e do VE"(16). "Na IMi aguda, a súbita sobrecarga de volume imposta ao $\mathrm{AE}$, sobreposta à incapacidade do AE e do VE dilatarem-se repentinamente, determinam a elevação da pressão venosa pulmonar ocasionando congestão pulmonar, dispnéia e freqüentemente edema agudo de pulmão $0^{(15-16)}$, podendo evoluir para choque cardiogênico"(17). "As repercussões clínicas na IMi crônica ocorrem tardiamente, ao compararmos com a EMi, sendo típico um longo período assintomático. Apesar de tardia, estas podem evoluir gradativamente, em decorrência das alterações hemodinâmicas que contribuem para a deterioração silenciosa da função ventricular esquerda"(17).

\section{METODOLOGIA}

0 trabalho* foi desenvolvido no Ambulatório de Cardiologia do HC/UNICAMP, que organiza o atendimento aos pacientes, de acordo com as subespecialidades da Cardiologia. Sendo assim, os portadores de valvopatia são atendidos às sextas-feiras, no período da manhã.

A amostra do estudo foi constituída por 29 pacientes, portadores de estenose e/ou insuficiência mitral, atendidos no referido serviço, no período de novembro de 1997 a fevereiro de 1998, verbalmente comunicativos, maiores de dezoito anos e que concordaram (consentimento informado) em participar da pesquisa. Os dados foram obtidos através de entrevista com o paciente e consulta ao prontuário hospitalar para levantamento de dados clínicos pertinentes.

Para verificar a validade do instrumento de coleta de dados, foi realizado um teste piloto envolvendo dez pacientes, procedendose a algumas alterações necessárias à sua melhor adequação.

Os itens fechados foram analisados de modo quantitativo e seus resultados expressos em números absolutos e percentuais; as questões abertas foram analisadas utilizando-se o método de Análise de Conteúdo ${ }^{(1)}$. Este tipo de análise tem por objetivo "compreender criticamente o sentido das comunicações, seu conteúdo manifesto ou latente, as significações explícitas ou ocultas"(18).

\section{RESULTADOS E DISCUSSÃO}

Em relação à caracterização do grupo estudado, encontramos predomínio do sexo feminino $(93,1 \%)$, com a idade média de 40,3 anos (21 a 59 anos). A idade na qual surgem os sintomas de distúrbio valvar pode ser um indicativo da natureza do

\footnotetext{
* Projeto de Pesquisa aprovado pelo Comitê de Ética da Faculdade de Ciências Médicas da UNICAMP, em cumprimento à Resolução 196/96, do Conselho Nacional de Saúde (diário oficial da União - Ministério da Saúde, 23.9.1997)
} 
distúrbio ${ }^{(19)}$. Dessa forma, quando o indivíduo apresenta idade inferior a 30 anos, a causa é provavelmente congênita; na faixa de idade de 30-70 anos, a valvopatia é provavelmente devida à doença cardíaca reumática, que afeta mais freqüentemente a valva mitral. Acima dos 70 anos a causa mais freqüente é a calcificação dos folhetos valvares. Antecedentes de febre reumática foram relatados por 16 pacientes $(55,2 \%)$, dados corroborados pela consulta ao prontuário.

Quanto à procedência, 17 pacientes $(58,6 \%)$ eram da cidade de Campinas e região administrativa e $12(41,4 \%)$ procedentes de outras localidades. A maioria dos pacientes $(79,3 \%)$ possuía o primeiro grau incompleto, $10,3 \%$ eram analfabetos, $6,9 \%$ possuíam primeiro grau completo e apenas $3,4 \%$ apresentavam 0 segundo grau completo. A renda familiar apresentou significativa concentração (44,8\%) na faixa de 3-6 salários-mínimos; 37,9\% informaram renda inferior a três salários-mínimos; $10,3 \%$ entre 610 salários-mínimos e 6,9\% superior a 10 salários-mínimos.

Com relação à ocupação, a maioria $(79,3 \%)$ apresentava algum tipo de atividade profissional, previamente ao diagnóstico de valvopatia e 6 pacientes (20,7\%) eram inativos, porém verificou-se que houve um aumento do número de pacientes que deixaram de exercer a sua atividade profissional após o diagnóstico da valvopatia, o que implicou no maior número de pessoas na categoria dos que não trabalham (de 20,7 para 58,6\%).

Quanto ao diagnóstico da doença valvar, 14 pacientes $(48,3 \%)$ possuíam dupla lesão (estenose e insuficiência mitral); 9 (31\%) possuíam insuficiência mitral e 6 (20,7\%), estenose mitral.

Com relação ao tipo de tratamento realizado, 15 pacientes $(51,7 \%)$ encontravam-se em tratamento clínico, porém já tendo realizado algum tipo de tratamento cirúrgico e 14 pacientes $(48,3 \%)$ encontravam-se apenas sob tratamento clínico.

Dos que se submeteram a algum tipo de intervenção cirúrgica, cinco $(33,2 \%)$ tinham realizado comissurotomia e plastia mitral simultaneamente; quatro pacientes $(26,7 \%)$ submetidos somente a comissurotomia mitral; quatro $(26,7 \%)$, plastia mitral; um $(6,7 \%)$ troca de válvula mitral, não havendo registro sobre 0 tipo de procedimento a que havia sido submetido um paciente (6,7\%). Destacamos, que a maior parte das intervenções cirúrgicas foram realizadas após o ano de 1990 (86,7\%).

Ao avaliarmos o conhecimento dos pacientes sobre sua doença, constatamos que todos os entrevistados citaram aspectos relacionados à categoria denominada de representação de sua doença, como mostra a Tabela 1.

A representação da doença refere-se ao modo como 0 sujeito sente, percebe e/ou define o que acontece consigo. Estas definições são diferentes, mas nota-se que há semelhanças entre algumas. Neste sentido, a maioria dos pacientes $(93,1 \%)$ percebe o coração funcionando como uma máquina, que possui uma função mecânica e que, se comprometida, acarretará o mau funcionamento do órgão. Portanto, estes pacientes trazem no seu discurso o termo "entupimento da válvula do coração" o qual compromete a circulação do sangue.

Outros definem a doença em termos da forma como é tratada. Assim, referem-se ao tratamento, principalmente à intervenção cirúrgica, salientando a necessidade ou não de troca da válvula comprometida. Com menor freqüência, surge a questão da repercussão da doença em suas vidas. Estes pacientes relacionam a doença à sintomatologia causada pela doença, que por sua vez interfere no seu estilo de vida.

Tabela 1 - Distribuição da freqüência da população estudada, quanto ao conhecimento sobre sua doença, segundo as categorias temáticas, com exemplos, Campinas-SP, 1998

\section{CATEGORIÁS TEMÁTICAS}

$\mathrm{FA}^{1} \quad \mathrm{FR}^{2}$

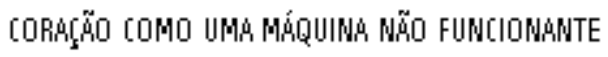

... hí 17 anos que tenho velo encpida. O D. Disse que

os exames mostraram veiz entpida...

... tentro problems na valivida. Em vez dejogar o sangue

para fora, joga para dento...

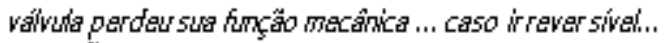

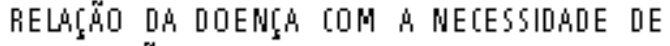

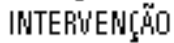

... tithh que fazer cirurgia. Foi faita correçäo, näo foi trocada a valivia...

... há muthos anos quando estava com problema, fir

$12 \quad 41,4$ operada (pus uma valkuta mitrail...

REPERCUSSÄ̃O DÁ DOENÇA NÁ VIDÁ DO SUJEITO

... ai dava falta de ar, näo dava para andar, trabalhar...

$\begin{array}{llll}\text {... chegou a sair sangue na boca quendo fiquai grávida. } & 7 & 24,1\end{array}$

Fiqueri com gravidez de silto risco...

INCAPACIDAODE DE DEFINIR O QUUE TEM

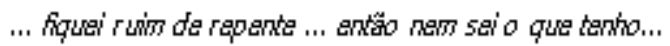
... näo sei dirato se é um sopro no coraçăo, fabre $2 \quad 6,9$ reumsatica...

${ }^{1}$ Freqüência absoluta ${ }^{2}$ Freqüência relativa: do $n^{0}$ de $F A$, em relação aos 29 pacientes

Obs: Cada paciente pode ter apontado mais de uma categoria

Ao indagarmos sobre as orientações recebidas sobre a doença, 19 pacientes $(65,5 \%)$ do total de entrevistados informaram ter recebido orientações específicas sobre sua doença. Dentre as orientações mais apontadas destacou-se a categoria que denominamos de cuidados decorrentes da existência da doença (Tabela 2). 
Tabela 2 - Distribuição da freqüência das respostas dos 19 pacientes que receberam orientações sobe sua doença, segundo as categorias temáticas, com exemplos, Campinas-SP, 1998

CATEGORIASS TEMÁTICASS

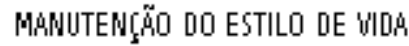

... pode ter vida normal...

... pode comer e fazer de twdo (até car pir)...

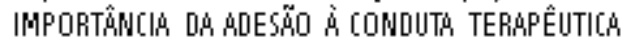

... continuar o thatamento porque daqui' 1 a 2 anos praciso

fazer outha cir urgia...

... tomar certo os madicamentos. Se demorar muito a $15 \quad 78,9$

cirurgia, uma das partas que están inchada pode atus sobre

soutra. Aio músculo pode ser afatado...

CUIDÁDOS DECORRENTES DÁ EXISTÊNCIŔ DÁ DOENÇA

... חäo fazer munto esforço ... me cuñdar durante 1 ano

para näo correr o risco de engravidar de novo...

... dentes am dia pra evitar complïaŗäo...

${ }^{1}$ Frequeência absoluta ${ }^{2}$ Frequêencia relativa: do n ${ }^{0}$ de $F A$, em relação aos 29 pacientes Obs: Cada paciente pode ter apontado mais de uma categoria

Em relação ao profissional que forneceu estas orientações, verificamos que a totalidade dos pacientes foram orientados pelos médicos, o que nos mostra a necessidade de uma atuação mais efetiva do enfermeiro na educação de pacientes portadores de valvopatias.

Para que a atuação de enfermagem seja efetiva, junto aos pacientes portadores de distúrbios valvares, é necessário que 0 profissional procure adotar uma abordagem de saúde mais abrangente, que envolva a perspectiva do paciente sobre sua saúde. Buscar a compreensão do que significa para o indivíduo ter uma doença valvar e conhecer como ele e sua família respondem à doença, como sentem, abordam e adaptam-se aos sintomas, constitui uma visão profunda do cuidado de enfermagem a pacientes valvopatas $^{(19)}$.

Portanto, a questão do que significa viver com uma valvopatia, as mudanças que a doença provavelmente acarreta no estilo de vida de seus portadores e, conseqüentemente, os mecanismos de enfrentamento lançados pelos indivíduos que vivem estas mudanças, devem fazer parte das investigações de enfermagem destinadas à melhoria da qualidade da assistência prestada a estes pacientes.

Ao questionarmos se as orientações fornecidas eram seguidas pelos pacientes, verificamos que dos 19 pacientes $(65,5 \%)$ que receberam informações, $17(89,5 \%)$ responderam que seguiam-nas. Os dois pacientes $(10,5 \%)$ que relataram não seguir as recomendações, referiram que a falta de adesão devia-se às condições de trabalho que, em parte, envolviam a realização de atividades com demanda de esforço físico, os colocava em desacordo com a suposta recomendação médica de evitar esforço físico excessivo.

Ao avaliarmos o desejo do grupo estudado em receber outras orientações sobre a doença e/ou tratamento, verificamos que 24 pacientes $(82,8 \%)$ gostariam de receber informações adicionais sobre sua doença e tratamento, de acordo com as categorias mostradas na Tabela 3.

Tabela 3 - Distribuição da freqüência dos 24 pacientes que especificaram quais as orientaç̃oes que gostariam de receber sobre sua doença ou tratamento, segundo as categorias temáticas, com exemplos, Campinas-SP, 1998

CATEGORIASS TEMÁTICASS $\mathrm{FA}^{1}{ }^{1} \mathrm{FR}^{2}$

ADIÇ̌̃̃ DE ELEMENTOS PäRA CONSTRUIR MELHOR SUÁ

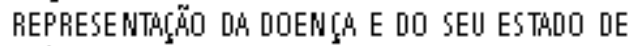
SBÚDE

... por qua tenho esse probima? Como começa a doença? $E$ assa dor que sinto? A doença á hereditarilia?...

... o que éso certo minha doença...

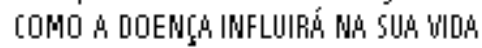

... o que posso fazer de atividade porque näo sei ficar

parada ... se en thabalhar numa fábrica, será qua se eur esforçasse au poderia ter probiama?...

$10 \quad 41,7$

... o qua posso comer? Como de tho mas acho que

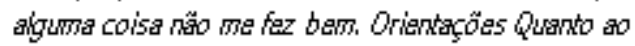
eforço...

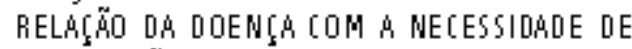

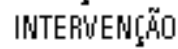

... sobre a cir urgia que provavelmente vou fazer...

... detalhadamente daqui a Quanto tempo vou ter que operar...

RELAÇÃO DA DOENÇA COM Á NECESSIDÁDE DE

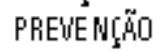

... cundados para prevenir...

... como prevenir para evitar de Jer novos casos na $2 \quad \begin{array}{lll}8 & 3\end{array}$ familia...

${ }^{1}$ Freqüência absoluta ${ }^{2}$ Freqüência relativa: do n ${ }^{0}$ de $F A$, em relação aos 29 pacientes Obs: Cada paciente pode ter apontado mais de uma categoria

No planejamento das atividades educativas, a serem realizadas com a clientela, é essencial que os profissionais considerem as características da população (culturais, socioeconômicas) e suas necessidades. É fundamental que a construção do conhecimento nas práticas educativas se dê a partir daquilo que os pacientes já sabem, constituindo-se numa relação de troca entre profissionais/clientela, o que pode facilitar a formação de vínculos ${ }^{(20)}$.

A despeito do conhecimento que o enfermeiro traz em sua bagagem - do que seja a doença, quais são suas repercussões clínicas e alguns cuidados básicos que recomenda-se sejam tomados pelos indivíduos acometidos por esta ou outra afecção - é imperativo que se busque conhecer o que o indivíduo reconhece como importante para que possa, a partir da construção de um novo conhecimento, redirecionar sua vida. Assim, no grupo estudado, constatamos a necessidade de conhecimento sobre a própria doença e principalmente, o modo como ela implicará em sua vida. 0 
aparecimento desta questão direciona o enfermeiro a uma nova busca, a de conhecimento em maior profundidade do estilo de vida de cada indivíduo, pois o reconhecimento das implicações da doença sobre a vida do sujeito, só é possível a partir da constatação de como a pessoa vive.

Dentre os cinco pacientes $(17,2 \%)$ que referiram preferir não receber orientações adicionais sobre sua doença e tratamento, constatamos que os aspectos relacionados à afetividade foram os mais significativos, como mostra a Tabela 4.

Tabela 4 - Distribuição da freqüência dos 5 pacientes que não desejavam orientações adicionais sobre sua doença ou tratamento, segundo as categorias temáticas, com exemplos, Campinas-SP, 1998

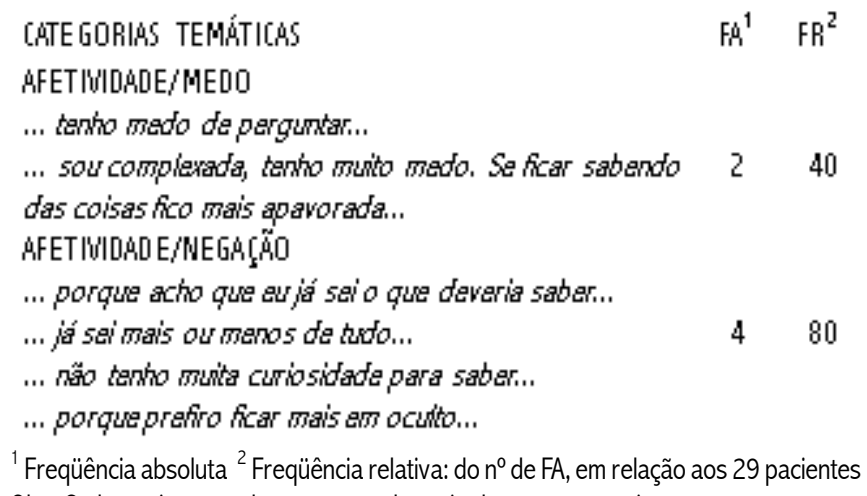

"Há no inconsciente de todo ser humano terror à aniquilação da vida, denominada de angústia primária, cuja origem é a percepção da atuação do instinto de morte no organismo"(21). Portanto, diante de uma situação que envolve ameaça à vida a pessoa experimenta um sentimento de angústia que dependendo da magnitude, poderá desencadear reações protetoras ou, quando em excesso levar a uma paralisação frente à situação vivida. Dessa forma, o paciente que sofre de cardiopatia é constantemente colocado em confronto com a intensificação da angústia de morte, uma vez que o "coração representa o motor da vida" podendo levar à mobilização de intensas defesas (entre elas a negação da doença) que visam a proteção contra a angústia aniquiladora. Podem ocorrer várias possibilidades de reações emocionais, sendo que aqueles que toleram a realidade da constatação da cardiopatia, terão que se adaptar aos novos limites impostos pela doença havendo, portanto, sempre algum grau de ajuste emocional.

Em relação, especificamente, aos pacientes portadores de valvopatia, a necessidade de entrar em contato com a doença, comprometer-se com o tratamento e a possibilidade de submeterse a uma intervenção cirúrgica torna o paciente valvopata temeroso, sendo incontestável a mobilização de profundas emoções, acompanhada pela negação da doença. Portanto, é recomendado um acompanhamento psicoterápico, principalmente para aqueles que irão submeter-se a uma intervenção cirúrgica, para auxiliar na adaptação à nova situação(22)
Em relação à alteração no estilo de vida em decorrência da presença da valvopatia, encontramos no grupo estudado 23 pacientes $(79,3 \%)$ que referiram modificação em suas vidas em decorrência da doença. A categorização das modificações relatadas por estes pacientes está colocada na Tabela 5.

Tabela 5 - Distribuição da freqüência dos 23 pacientes que citam as modificações em suas vidas em decorrência da valvopatia, segundo as categorias temáticas, com exemplos, Campinas-SP, 1998

$$
\text { CATEGORIAS TEMÁTICASS } \mathrm{FA}^{1}{ }^{1} \mathrm{FR}^{2}
$$

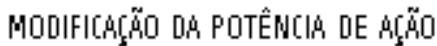$$
\text { ... mudouro jaito de viver, näo é mais como antes, näo }
$$$$
\text { posso fazer esforços, estou mais lïmitada... }
$$$$
16 \quad 69,6
$$$$
\text { ...dissinui minha atividade, sinto-me libsitada por se }
$$$$
\text { portadora de uma doença. }
$$$$
\text { MUDÁNÇA NA AFETIVIDÄDE }
$$$$
\text { ... psicologicamente, munto ner vosismo, chorosa, fiquai, }
$$$$
\text { tornai-me uma pessoa carente, parece que virou }
$$$$
\text { depressäo... }
$$

... angústia, näo somos mais como as outras pessoas...

... frzo as coisas semprecom mado...

... חăo sinto ânimo para nada...

${ }^{1}$ Freqüência absoluta ${ }^{2}$ Freqüência relativa: do n ${ }^{0}$ de FA, em relação aos 29 pacientes Obs: Cada paciente pode ter apontado mais de uma categoria

Quanto à categoria modificação da potência de ação do total de 23 pacientes entrevistados, $65 \%$ apresentavam modificação em suas vidas, percebendo-se mudanças de comportamento, principalmente com relação ao trabalho e às atividades físicas. Os dados mostraram nitidamente o reconhecimento da limitação causada pela valvopatia ${ }^{(7)}$.

Destaca-se, no entanto, o número significativo de pacientes, em nosso estudo $(47,8 \%)$ que referiram alterações no âmbito da afetividade, o que mostra a importância das modificações em nível emocional sofridas pelos pacientes valvopatas.

Convém ressaltar que as alterações relacionadas à categoria afetividade podem ainda ser explicadas, pelo fato de um grande número de pacientes desta população $(51,7 \%)$ ter sofrido algum tipo de intervenção cirúrgica.

0 ajustamento psicológico do indivíduo após uma intervenção cirúrgica cardíaca é lento e difícil. Em um estudo que envolveu 118 pacientes submetidos a tratamento cirúrgico, avaliados um ano após a intervenção, foi observado que em conjunto com a melhora das condições físicas proporcionadas pela intervenção cirúrgica houve melhora relacionada a fatores psicológicos em 75\% dos pacientes. Porém, $25 \%$ dos pacientes evoluíram com persistência e/ou surgimento de novos fatores psicológicos considerados geradores de estresse. Dessa forma, a qualidade de vida de uma parcela destes pacientes permaneceu similar àquela que possuíam previamente ao procedimento cirúrgico ${ }^{(23)}$.

Os pacientes cardiopatas são colocados em confronto com 
uma intensificação da angústia de morte, uma vez que o coração "representa o motor da vida"(21).

Em estudo comparativo ${ }^{(22)}$ com o objetivo de avaliar as reações emocionais e as alterações da imagem corporal, da autoestima e autoconceito em pacientes coronariopatas e valvopatas que aguardavam tratamento cirúrgico através de entrevista e o emprego da técnica de desenho da Figura Humana de Machover, os pacientes de ambos os grupos apresentavam dificuldade para lidar com mudanças no estilo de vida, causadas pela doença e tratamento.

Tais dados reforçam a importância de considerar, no planejamento da assistência de enfermagem, uma abordagem que contemple os aspectos fisiológicos, psicológicos e sociais do paciente valvopata.

A associação do conhecimento da fisiopatologia da doença, com a compreensão de como o paciente e família percebem a situação de doença, contribuirá para que o enfermeiro e/ou a equipe de saúde possam ajudá-los na adaptação às mudanças impostas pela valvopatia, independente do tipo de tratamento realizado (clínico e/ou cirúrgico).

Ao avaliarmos os dados referentes à modificação na vida após tratamento da doença, verificamos que dos 22 pacientes que referiram modificação em suas vidas, a maioria $(77,3 \%)$ citou aspectos relacionados à modificação da sintomatologia, referindo em suas falas uma melhora em seu quadro clínico (Tabela 6).

Tabela 6 - Distribuição da freqüência dos 22 pacientes que citaram as modificações em suas vidas após o tratamento da doença, segundo as categorias temáticas, com exemplos, Campinas-SP, 1998

\section{CATEGORIBSS TEMÁTICAS}

MODIFICAÇÕES RELACIONADÁS \&̆ SINTOMATOLOGIÁ

... a cirurgia musou para malhor a näo me sinto como

antes: falla de ar, peso do irdo esquerdo, cansarco...

... antes, precisava sentar vórias vezes para varrer. Agora

näo preciso mais. Depois do tratamento mádico, mellhorei

donervoso...

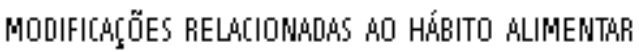

... ankes näo me änmentava. Agora, estoume ämmentando

mellhor...

... engordaiseis quinos...

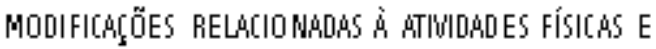

DE TRḾB BQ́LLHO

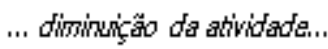

... parei de trabathar, aposentai...

TOTALL

A Tabela 7, a seguir, mostra que, dentre os sete pacientes $(24,1 \%)$ que referiram não haver nenhuma modificação em suas vidas após o tratamento médico, os motivos mais citados para tal

relato foram: a inalteração nas atividades de trabalho e inalteração na sintomatologia, ambos com percentual de 42,9\%.

Tabela 7 - Distribuição da freqüência dos 7 pacientes que negaram modificação em suas vidas após o tratamento da doença, segundo as categorias temáticas, com exemplos, Campinas-SP, 1998

CÄTEGORIÁS TEMÁTICASS $\mathrm{FH}^{1} \quad F \mathrm{R}^{2}$

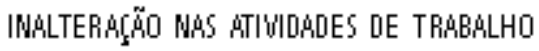

... thaballho normalmente..

... sampre trabahei, so que agora me sinto mais

cansads...

$3 \quad 42,9$

... frour do mesmo jaito. Emprego á coisa difill de

arrumer...

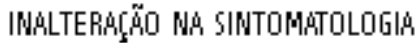

... contiwo com dor, se euficar samo ramádo, voltam

os sintomas.

... mellhorou os sintomas após a cirurgiá, mas agora $3 \quad 42,9$ estú voltando...

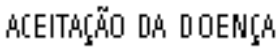

... porqua para mins foi normal

$1 \quad 14,3$

TRMTMMMENTO RECENTE

... anda está recente... estou ainds amedrondads com $\quad 1 \quad 14,3$

a volta dos sintomas...

${ }^{1}$ Frequiência absoluta ${ }^{2}$ Frequêencia relativa: do n ${ }^{0}$ de $F A$, em relação aos 29 pacientes Obs: Cada paciente pode ter apontado mais de uma categoria

Em relação às limitações para realizar atividades de trabalho, domésticas ou de lazer, 25 pacientes $(86,2 \%)$ responderam sentir-se limitados no desempenho destas atividades. As atividades nas quais sentiram-se mais limitados foram relacionadas à atividade física, como mostra a Tabela 8.

Tabela 8 - Distribuição da freqüência das respostas dos 25 pacientes em relação às atividades que acarretam limitações, segundo as categorias temáticas, com exemplos, Campinas-SP, 1998

CATEGORIMS TEMÁTICASS

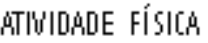

... andar muto tanto no plano como na subida...

... subir morro...

20

... correr... andar depressa...

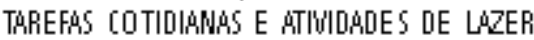

... esfiggar o banhairo, lavar o Qunxal..

... กäo posso mais apitar jogo de fitabol..

... pega peso...

${ }^{1}$ Frequeência absoluta ${ }^{2}$ Frequêencia relativa: do n ${ }^{0}$ de $F A$, em relação aos 29 pacientes Obs: Cada paciente pode ter apontado mais de uma categoria

As limitações citadas pelos pacientes, tanto para 0 desenvolvimento das atividades físicas como para as cotidianas, incluindo as atividades domésticas e de lazer, relacionaram-se à presença de sintomatologia como: taquicardia, dispnéia, fadiga, sudorese, dor torácica, escurecimento da visão, extremidades frias e enfraquecimento das pernas.

Os resultados descritos acima nos levam a considerar que estas limitações possivelmente prejudicam o desenvolvimento de 
atividades físicas do tipo aeróbica regular (nadar, caminhar, pedalar, etc.). Dessa forma, 22 pacientes $(75,9 \%$ ) não desenvolviam qualquer atividade física deste tipo. Dos sete pacientes $(24,1 \%)$ que realizavam atividade aeróbica, aquela que mais se destacou foi a caminhada, citada por quatro pacientes.

As limitações impostas pela doença levam à diminuição de atividades físicas diárias ${ }^{(7)}$. Os pacientes valvopatas têm dificuldades em monitorizar e reconhecer os sinais de fadiga principalmente no início da doença, além de tender a subestimar a energia requerida pelas atividades diárias, e acrescenta que o problema na verdade pode estar não no estabelecimento de limites, mas ligado ao sentimento de invalidez decorrente do "estar doente" ${ }^{\text {"(19) }}$.

Dados recentes, oriundos do European Heart Failure Training Group ${ }^{(24)}$, demonstraram, ao contrário, que pacientes com Insuficiência Cardíaca Congestiva, classificação funcional I, II e III, desde que estáveis, podem obter benefícios substanciais a partir de programas de condicionamento, nos quais são submetidos a sessões freqüentes e regulares de atividade aeróbica, por determinado período de tempo, sem maiores efeitos colaterais. Um aumento, mesmo que modesto, em sua capacidade de realizar exercício pode melhorar significativamente sua capacidade para tarefas diárias.

0 consenso sobre conduta em valvopatia, publicado em $1996^{(8)}$, propõe a introdução lenta e progressiva da atividade física após atingir-se a estabilidade clínica. Vale destacar que durante a fase de descompensação, o repouso é recomendado. A característica do exercício a ser prescrito, segundo os autores desse consenso, estaria pautada na avaliação da área valvar, rítmo cardíaco e pressão de pico na artéria pulmonar, em repouso ou exercício. Assim, a presença de área valvar menor que $1,4 \mathrm{~cm}^{2}$, alteração do ritmo cardíaco (com fibrilação atrial) e do pico de pressão na artéria pulmonar maior que $50 \mathrm{mmHg}$ como fatores isolados ou concomitantes levam à indicação de alguma limitação da atividade física, embora a proibição irrestrita seja indicada apenas nos casos em que a área valvar é menor que $1,1 \mathrm{~cm}^{2}$ ou encontra-se entre 1,1 e $1,4 \mathrm{~cm}^{2}$, porém associada a alteração do ritmo cardíaco e pressão de pico na artéria pulmonar maior que $80 \mathrm{mmHg}$ durante 0 exercício, no caso da estenose mitral. Na insuficiência mitral, a proibição baseia-se no rítmo cardíaco, tamanho e função ventricular.

Utilizando os resultados do ecocardiograma e do cateterismo cardíaco (últimos resultados registrados no prontuário hospitalar) dos pacientes que compuseram a amostra deste estudo, realizamos a distribuição destes, segundo as recomendações sobre prática de atividade esportiva proposta pelo consenso acima citado $^{(8)}$, como mostram as Tabelas 9 e 10.

Observa-se nas Tabelas 9 e 10 que um grande número de pacientes ${ }^{(17)}$ possuía permissão irrestrita para o exercício de atividade física e que, dentre os demais, apenas dois teriam proibição irrestrita.
Tabela 9 - Distribuição dos 17 pacientes com diagnóstico de estenose mitral $^{*}$, de acordo com a prática esportiva mais indicada ${ }^{(8)}$. Campinas-SP, 1998

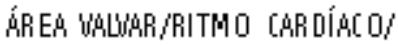

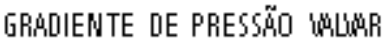

uM $\neq \neq>1,5 \mathrm{~cm}^{2}$, ritmo sinusal, pressão de pico na artéria pulmonar $<50 \mathrm{mmHg}$.

M⿻川 $M>1,5 \mathrm{~cm}^{2}$, em fibrilação atial, pressão de pico na artéria pulmonar $<50 \mathrm{~mm} H \mathrm{Hg}$ emrepouso ou em exercício.

HulM $>1,5 \mathrm{~cm}^{2}$, em ritmo sinusal ou fibribção atrial, pressão de pico na artéria pulmonar de 50-80 $\mathrm{mmHg}$ em repouso ou em exerócio.

HalM $>1,1-1,4 \mathrm{~cm}^{2}$ em ritmo sinusal ou fibribção atrial, pressão de pico na artéria pulmonar $<50 \mathrm{mmHg}$ em repouso ou em exercício.

uM $>1,1-1,4 \mathrm{~cm}^{2}$ em ritmo sinusal oufibribção atrial , pressão de pico na artéria pulmonar de 50-80 $\mathrm{mmHg}$.

M.M $M>1,1-1,4 \mathrm{~cm}^{2}$ em ritmo sinusal ou fibribção atrial, pressão de pico na artéria pulmonar $>80 \mathrm{mmHg}$ durante exercício.

$\mathrm{AM}<<1,1 \mathrm{~cm}^{2}$

TOTÁL

N $\%$

Permissão irrestrita $\quad 8 \quad 47$

Permissão para

atividadeisométrica

baixae moderadae

$2 \quad 11,8$

isotônica baixa.

Permissão para

atividade

$4 \quad 23,5$

Isométrica baixa e

moderada e

isotônica baixa.

Permissãopara ati-

vidade isométrica

$1 \quad 5,9$

baixae moderadae

isotônica modera-

da.

Permissão para

atividadeisométrica

baixae moderadae

isotônica baixa.

211,8

Proibição irrestrita

Proibição irrestrita

$17 \quad 100$

* Foram considerados os pacientes com diagnóstico predominante de Estenose Mitral

** Área valvar mitral (AVM)

Tabela 10 - Distribuição dos 11 pacientes com insuficiência mitral* quanto às recomendações sobre prática de atividade esportiva, de acordo com função ventricular esquerda e rítmo cardíaco**

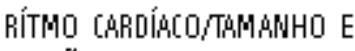
FUNÇ,

Rítmo sinusal com tamanho e Permissão irrestrita. $\quad \begin{array}{lll}9 & 81,8\end{array}$ funçãa $\mathrm{VE}^{\neq \neq \neq}$normais.

Ritmo sinusal ou fibrilação atrial Permissão para ativicom aumento WE discreto e dade isométrica baixa funç̧̃̃o normal. e moderada e isotônica baixa e moderada.

Aumento VE definido ou qualquer Proibição irrestrita grau de diffunção VE em repouso.

TOTÁL

$11 \quad 100$
* Foram considerados os pacientes com diagnóstico predominante de Insuficiência Mitral

**.. de acordo com o consenso sobre conduta em valvopatia ${ }^{(8)}$

***VE (ventrículo esquerdo)

$\mathrm{N}_{1}$ : número de indivíduos da população estudada, incluídos nessa categoria

$\mathrm{N}_{2}$ : número de indivíduos da categoria que efetivamente realizavem a atividade permitida 
Ao analisarmos a informação sobre a atividade sexual dos pacientes, encontramos que do total de pacientes, seis $(20,7 \%)$ negaram possuir atividade sexual. Aqueles que possuíam atividade sexual foram indagados quanto à presença de limitações durante a relação sexual. Constatamos que dos 23 pacientes que possuíam atividade sexual, 17 (73,9\%) apresentavam limitações no intercurso das relações, que foram atribuídas à sintomatologia decorrente da doença sendo citado principalmente, o cansaço e a dispnéia (52,9 e $64,7 \%$, respectivamente).

\section{CONCLUSÕES}

No grupo estudado predominou o sexo feminino, com idade média de 40,3 anos, em sua maioria procedentes da cidade de Campinas-SP, com primeiro grau incompleto e que possuíam atividade profissional antes da manifestação da doença. Quanto ao tratamento médico da valvopatia, $51,7 \%$ dos pacientes haviam sido submetidos à cirurgia e encontravam-se em tratamento clínico, junto com os demais.

Os pacientes estudados demonstraram conhecimento sobre a doença, percebendo-a pelo funcionamento do coração, forma de tratamento e repercussão da doença em suas vidas.

A maioria dos pacientes $(89,5 \%)$ reportou adesão ao tratamento e gostaria de receber mais informações sobre a doença e tratamento.

\section{REFERÊNCIAS BIBLIOGRÁFICAS}

1. Bardin L. Análise de conteúdo. Lisboa (Portugal): Edições 70; 1977.

2. Lotufo PA. Epidemiologia das doenças cardíacas no Brasil: histórico, situação atual e proposta de modelo teórico. RSCESP 1996; 6(5):541-7.

3. Lotufo PA, Lolio CA. Tendências de evolução da mortalidade por doenças cardiovasculares: o caso do Estado de São Paulo. In: Monteiro CA, organizador. Velhos e novos males da saúde no Brasil: a evolução do país e de suas doenças. São Paulo (SP): HucitecNUPENS/USP; 1995.

4. Gus I, Zaslavsky C, Seger JMP, Machado RS. Epidemiologia da febre reumática: estudo local. Arq Bras Cardiol 1995; 65(4):321-5. 5. Eisenberg MJ. Rheumatic heart disease in the developing world: prevalence, prevention and control. Eur Heart J 1993; 14:122-8.

6. Vasan RS, Shirivastava S, Vijayakumar M, Narang R, Lister BC, Narula J. Echocardiographic evaluation of patients with acute rheumatic fever and rheumatic carditis. Circulation 1996; 94(1):7382.
A valvopatia modificou o estilo de vida da maioria dos pacientes, em geral, com repercussões negativas, principalmente no âmbito da afetividade e de atividades físicas, atenuadas após a instituição do tratamento.

\section{CONSIDERAÇÕES FINAIS}

Apesar da literatura sobre a assistência de enfermagem ao paciente portador de valvopatia mitral voltar-se, em especial, aos cuidados na fase aguda da doença é necessário considerar as profundas modificações por ela imposta, a longo prazo, na vida destas pessoas.

As mudanças no estilo de vida a partir de determinada fase da doença são tão intensas, que demandam; por parte daqueles que com ela convivem, o desenvolvimento de mecanismos de adaptação biológica, psicológica e social.

Sendo assim, procurar identificar junto ao paciente valvopata, o que sabe sobre sua doença, o que para ele significa adoecer do coração, qual a sua perspectiva e de sua família sobre o problema e, principalmente, como ele convive com as novas condições impostas pela doença é fundamental para elaboração de qualquer estratégia educacional, que vise atingir o paciente na comunidade e, desta forma contribuir para a manutenção ou melhoria de sua qualidade de vida.

7. Guimarães GCQCP, Vianna LAC. Características do paciente valvopata com diagnóstico de excesso de líquido e déficit de conhecimento sobre a doença. RSCESP 1993; 3(4 Supll A):5-8.

8. Grinberg M, coordenador Consenso sobre conduta em valvopatia. RSCESP 1996; 6(3):378-90.

9. Décourt LV, Pileggi F. organizadores Valvopatias cardíacas. In: Gonçalves EL et al, editores. Manual de clínica médica. Rio de Janeiro (RJ): Guanabara Koogan, 1980. p.755-65.

10. Carulla VF. Enfermidades do coração. In: Domarus, AV Medicina interna. 10 a ed. Rio de Janeiro (RJ): Guanabara Koogan; 1985. p.466-500.

11. Carter L, Lamerton, M. Understanding balloon mitral valvuloplasty: the Inoue technique. Intensive Critical Care Nurs 1996; 12:147-54.

12. Braunwald E. Valvular heart disease. In: Braunwald, E. Heart disease: a textbook of cardiovascular medicine. $4^{a}$ ed. Philadelphia: W. B. Saunders; 1992. p.1007-77.

13. Kosakai Y, Kawaguchi AT, Isobe F, Sasako Y, Nakano K, Eishi K et al. Cox maze procedure for chronic atrial fibrillation associated with mitral valve disease. J Thorac Cardiovasc Surg 1994; 108(6):104955. 
14. Lin JM, Hsu KL, Hwang JJ, Li YH, Tseng YZ. Interference of mitral valve stenosis with left ventricular diastole and left atrial appendage flow. Cardiology 1996; 87:537-44.

15. Rackley CE. Valvulopatias cardíacas. In: Wyngaarden JB, Smith LH, editores. Tratado de medicina interna. $19^{a}$ ed. Rio de Janeiro (RJ): Guanabara Koogan; 1993. p.331-5.

16. Couto AA de, Oliveira GMMO, Costa CLP, Magalhães CK, Garce, CMF. Avanços em clínica cardiológica parte 3 - insuficiência mitral crônica. Arq Bras Med 1994; 68(5):299-301.

17. Dallan LAO, Oliveira SAO, Atik F, Abreu CACF, Dias AR, Jatene FB et al. Insuficiência valvar mitral isquêmica. Incidência, diagnóstico e tratamento cirúrgico. Arq Bras Cardiol 1994; 62(5):329-36.

18. Chizzotti A. Pesquisa em ciências humanas e sociais. São Paulo (SP): Ed. Cortez; 1991.

18. Vala J. Metodologia das ciências sociais. Lisboa (Portugal): Edições Afrontamento, 1986.
19. King KM. Valve disorder: theorical and practical nursing perspectives. CICN 1994;5(1):13-7.

20. Cocco MIM. Projeto: Práticas educativas em saúde- possibilidades na construção da cidadania. Projeto de pesquisa em andamento, sem financiamento, FCM-UNICAMP 2001.

21. Svartman B. Adoecer do coração: visão psicanalítica. RSCESP 1994; 4(1 Supll A):5-8.

22. Pires CA, Sharovsky LL, Romano BW. Coronariopatas e valvopatas: impacto emocional da cirurgia cardíaca. Estudo comparativo. RSCESP 1994; 4(5 Supll A):1-7.

23. Magni G, Unger HP, Valfre C, Polesel E, Cesari F, Rizzardo R et al. Psychosocial outcome one year after heart surgery: a prospective study. Arch Intern Med 1987; 147:473-7.

24. European Heart Failure Trainig Group. Experience from controled trials of physical training in chronic heart failure. Protocol and pacient factors in effectiveness in the improvement in exercise tolerance. Eur Heart J 1998; 19:466-75. 